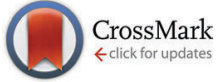

Cite this: Phys. Chem. Chem. Phys., 2016, 18, 28403

Received 16th June 2016 Accepted 20th September 2016

DOI: $10.1039 / c 6 c p 04217 a$

www.rsc.org/pccp

\section{Atomistic insights into deep eutectic electrolytes: the influence of urea on the electrolyte salt LiTFSI in view of electrochemical applications $\dagger$}

\author{
Volker Lesch, ${ }^{a}$ Andreas Heuer, ${ }^{\text {ab }}$ Babak R. Rad, ${ }^{a}$ Martin Winter ${ }^{\text {ac }}$ and Jens Smiatek ${ }^{{ }^{d}}$
}

The influence of urea on the conducting salt lithium bis-(trifluoromethanesulfonyl)-imide (LiTFSI) in terms of lithium ion coordination numbers and lithium ion transport properties is studied via atomistic molecular dynamics simulations. Our results indicate that the presence of urea favors the formation of a deep eutectic electrolyte with pronounced ion conductivities which can be explained by a competition between urea and TFSI in occupying the first coordination shell around lithium ions. All simulation findings verify that high urea concentrations lead to a significant increase of ionic diffusivities and an occurrence of relatively high lithium transference numbers in good agreement with experimental results. The outcomes of our study point at the possible application of deep eutectic electrolytes as ion conducting materials in lithium ion batteries.

Deep eutectic electrolytes (DEEs), also called deep eutectic solvents, are mixtures of salts with small polar organic molecules, which have a melting temperature lower than the melting temperatures of the constituting components. DEEs are nowadays used for synthesis purposes, separation techniques and also for electrochemical applications. ${ }^{1-3}$ Initially in 2003, Abbott et al. studied the prototype of a DEE by adding urea to choline chloride, ${ }^{4}$ which was later also investigated by molecular dynamics (MD) simulations. ${ }^{5}$ In contrast to most conventional organic solvents, choline based eutectic electrolytes are non-toxic, and have good biodegradability and low cost. Also, in terms of biochemical applications, the usage of DEEs might be a beneficial option to circumvent problems found for ordinary ILs. ${ }^{6,7}$ In addition, it was discussed that lithium based DEEs are potential candidates

\footnotetext{
${ }^{a}$ Helmholtz-Institute Muenster (IEK-12): Ionics in Energy Storage,

Forschungszentrum Juelich, Corrensstrasse 46, 48153 Muenster, Germany

${ }^{b}$ Institute of Physical Chemistry, University of Muenster, Corrensstrasse 28/30, 48149 Muenster, Germany

${ }^{c}$ MEET Battery Research Centre, Institute of Physical Chemistry, University of Muenster, Corrensstrasse 46, 48149 Muenster, Germany

${ }^{d}$ Institute for Computational Physics, University of Stuttgart, Allmandring 3, 70569 Stuttgart, Germany. E-mail: smiatek@icp.uni-stuttgart.de;

Fax: +49711685 63658; Tel: +4971163757

$\dagger$ Electronic supplementary information (ESI) available. See DOI: 10.1039/c6cp04217a
}

to replace IL/lithium salt mixtures in battery applications. ${ }^{8}$ In contrast to DEEs, ILs typically require a relatively large amount of dissolved lithium salt in order to be applicable for electrochemical devices with the drawback of high viscosities and low lithium ion conductivity. ${ }^{9-13}$ The main advantage of DEEs is the presence of a single cation species, for example $\mathrm{Li}^{+}$in LiTFSI, such that relatively high $\mathrm{Li}^{+}$transference numbers can be observed. ${ }^{8}$

A few years before the establishment of choline/urea DEEs, Liang et al. ${ }^{14}$ investigated the influence of urea on lithium bis(trifluoromethanesulfonyl)-imide (LiTFSI) in terms of electrochemical stabilities and lithium ion diffusivities. For specific molar ratios of $1: 3.6$ between LiTFSI and urea, ionic conductivities of around $10^{-4}$ to $10^{-3} \mathrm{~S} \mathrm{~cm}^{-1}$ at temperatures in the range from 300 to $330 \mathrm{~K}$ were reported. ${ }^{14}$ Furthermore, the eutectic melting temperature was found to be $240 \mathrm{~K}$ whereas LiTFSI and urea have individual melting temperatures of $507 \mathrm{~K}$ and $405 \mathrm{~K}$. First MD simulations revealed the presence of strong interactions between the oxygen atoms of urea and TFSI, which are mainly responsible for the observed effects. ${ }^{15}$ In addition, it was shown that mixtures of urea and LiTFSI are also applicable for polymer electrolyte systems ${ }^{16}$ and further results also revealed a similar behavior for LiTFSI/acetamide. ${ }^{17}$ Based on the experimental results, it can be concluded that the presence of urea weakens the interaction between the lithium and the TFSI ions but it yet has not been clarified in detail whether the lithium cation, the TFSI anion or both of them interact with urea and how the molecular composition arranges in contrast to the often studied interaction between urea and macromolecules. ${ }^{18-22}$ More recent technological applications of DEEs in the context of electrochemical devices include glycerol and choline iodide as electrolytes in solar cells ${ }^{23}$ and as solvents for the electrodeposition of zinc. ${ }^{24}$

Although the benefits of DEEs were often reported, ${ }^{2,3}$ atomistic insights into the underlying mechanisms that lead to the improvement of the mixtures with DEEs are sparse. A deeper understanding might stimulate the optimization of lithium transference numbers which is of prior importance for the application of DEEs in lithium based electrochemical devices. In order to study the interaction 
between urea and LiTFSI on the atomistic scale, we performed classical molecular dynamics simulations using the OPLS-AA ${ }^{25}$ force field. Our results indicate a subtle competing effect between urea and TFSI to occupy the coordination shell around lithium. The composition of the local environment strongly influences the dynamic properties of $\mathrm{Li}^{+}$such that high urea concentrations facilitate the occurrence of relatively high lithium transference numbers.

All molecular dynamics (MD) simulations were performed with the simulation package GROMACS (version 4.6.7). ${ }^{26-28}$ The starting structures of LiTFSI/urea mixtures were produced by PACKMOL. ${ }^{29,30}$ The force field parameters for LiTFSI were extracted from the OPLS-AA ${ }^{25}$ force field which was verified to reproduce structural properties for systems containing lithium salts in good agreement with the experimental results. ${ }^{31}$ In fact, classical atomistic force fields like OPLS-AA usually disregard the electronic properties of atoms which influences the dynamic behavior. ${ }^{32}$ Hence, it has to be noted that the obtained diffusion coefficients as well as the mass densities might vary quantitatively from experimental results. ${ }^{31,33,34}$ For a more reliable description in terms of exact values, the usage of polarizable atomistic force fields was proposed. ${ }^{32}$ Due to the absence of consistent polarizable force fields for the combination of urea and LiTFSI, we chose to study the properties of the system qualitatively in order to elucidate the main mechanisms in good agreement with the approach presented in ref. 33 and 34 . We simulated three different systems of LiTFSI and urea, namely mixtures of $300: 300: 300$ ions and molecules for urea $: \mathrm{Li}^{+}: \mathrm{TFSI}^{-}$ denoted as $\mathrm{U}_{1: 1}, 900: 300: 300\left(\mathrm{U}_{1: 3}\right)$ and 1800:300:300 $\left(\mathrm{U}_{1: 6}\right)$. The integration of Newton's equation of motion was performed by a Leapfrog algorithm. The elementary integration time step was $\delta t=2$ fs. The simulation time for all systems was $500 \mathrm{ns,}$ whereby the first 150 ns were used for equilibration and the remaining $350 \mathrm{~ns}$ were used for the analysis. We performed an energy minimization using the conjugate gradient method (energy tolerance: $100 \mathrm{~kJ} \mathrm{~mol}^{-1} \mathrm{~nm}$, step size: $10^{-4} \mathrm{~nm}$ ). The simulations were performed in NPT ensemble using the velocity rescale thermostat ${ }^{35}$ (coupling time: $1.0 \mathrm{ps}$ ) with a temperature of $T=400 \mathrm{~K}$ in combination with the Berendsen barostat ${ }^{36}$ (coupling time: $1.0 \mathrm{ps,} \mathrm{compressibility:} 10^{-6} \mathrm{bar}^{-1}$, reference pressure: 1 bar). All covalent bonds involving hydrogens were constrained by the LINCS algorithm. ${ }^{37}$ The electrostatic interactions were calculated using the smooth particle-mesh-Ewald method $^{38,39}$ with a cutoff range of $1.4 \mathrm{~nm}$ and an accuracy of $10^{-6}$.

We start the discussion with the dynamic properties of the species. The mean square displacement (MSD) of a species $\left\langle\left[\vec{r}_{i}(t)-\vec{r}_{i}\left(t_{0}\right)\right]^{2}\right\rangle$ is directly proportional to the diffusion coefficient $D_{i}$ via the Einstein relation ${ }^{40} D_{i}=\lim _{t \rightarrow \infty}\left\langle\left[\vec{r}_{i}(t)-\vec{r}_{i}\left(t_{0}\right)\right]^{2}\right\rangle / 6 t$ where $\vec{r}_{i}$ denotes a Cartesian coordinate at time $t$. Diffusive motion can be found for $\left\langle\left[\vec{r}_{i}(t)-\vec{r}_{i}\left(t_{0}\right)\right]^{2}\right\rangle \sim t^{\alpha}$ with $\alpha=1$ whereas subdiffusive motion is evident for $\alpha<1$. $^{33}$ The corresponding MSDs for lithium and TFSI in the presence of different urea concentrations are shown in Fig. 1 . It can be clearly seen that low urea concentrations $\left(\mathrm{U}_{1: 1}\right)$ hardly affect the dynamic
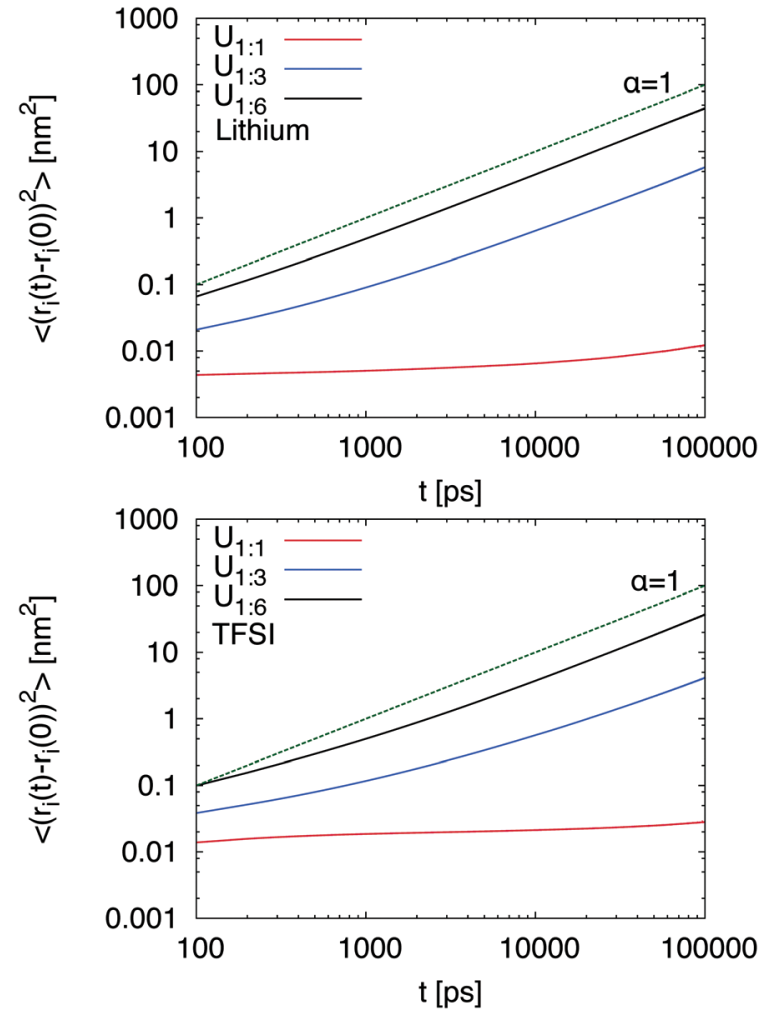

Fig. 1 Mean-square displacement (MSD) for lithium (top) and TFSI ions (bottom) under the influence of different urea concentrations. The dashed green line represents the theoretical result with $\alpha=1$ indicating the slope of the diffusive regime as a guide for the eye.

properties of lithium and TFSI ions. Hence, any motion is nearly absent and it can be concluded that the chosen urea concentration is too low to impose the formation of a DEE in agreement with experimental results. ${ }^{14}$ Thus, it can be assumed that a fully equilibrated structure even at an elevated temperature of $400 \mathrm{~K}$ is absent. Therefore, we will mainly focus on the results obtained for the systems $U_{1: 3}$ and $U_{1: 6}$ whereas the results for low urea content serve as a reference for the illustration of the main mechanisms. In contrast, higher urea concentrations $\left(U_{1: 3}\right.$ and $\left.U_{1: 6}\right)$ induce a diffusive behavior of lithium and TFSI ions already after $10 \mathrm{~ns}$. The corresponding transition times to the diffusive regime are comparable to alkylimidazolium based ionic liquids ${ }^{33,34}$ which points at the formation of a liquid DEE. The corresponding individual diffusion coefficients are presented in the ESI $\dagger$ where one can recognize the highest diffusivity for urea, followed by lithium and TFSI ions. The differences between lithium and TFSI are marginal with regard to the limited accuracy for dynamic properties obtained by non-polarizable force fields. ${ }^{41}$ Nevertheless, the small difference between lithium and TFSI diffusion coefficients stands in contrast to the behavior of LiTFSI in ionic liquids where the diffusion coefficient of TFSI is significantly higher. ${ }^{42}$ We will study this effect in more detail in the future.

After fitting the MSD to obtain the diffusion coefficients for all three species, we calculated the transference numbers for lithium ions. The lithium ion transference numbers 
give insights into the relative mobility of lithium ions in comparison to the other species in the system and are defined as $t_{\mathrm{Li}^{+}}=n_{\mathrm{Li}^{+}} D_{\mathrm{Li}^{+}} / \sum_{i} n_{i} D_{i}$ where $n_{i}$ denotes the number of ions or molecules of a species in the system. The resulting values are $t_{\mathrm{Li}^{+}}=0.09\left(\mathrm{U}_{1: 3}\right)$ and $t_{\mathrm{Li}^{+}}=0.07\left(\mathrm{U}_{1: 6}\right)$. In comparison with ILs, Lesch $e$ al. ${ }^{42,43}$ found a transference number of $t_{\mathrm{Li}^{+}}=0.039$ for 0.85 [emimTFSI] $/ 0.15[\mathrm{LiTFSI}]$ at $423 \mathrm{~K}$. Moreover, Ye et al. ${ }^{9}$ reported a transference number of $t_{\mathrm{Li}^{+}}=0.034$ at $293 \mathrm{~K}$ for a 1 M LiTFSI/P13TFSI/PVDF-HFP mixture. Thus, the slightly higher results for $t_{\mathrm{Li}^{+}}$in LiTFSI/urea mixtures indicate that the DEE seems to be a potential candidate for the usage as an ion conducting material in lithium based battery applications.

In order to achieve a consistent framework for the molecular composition of the DEE, we analyzed the local environment around lithium and TFSI ions in terms of radial distribution functions (RDFs) $g_{\alpha \beta}(r)$ and coordination numbers $\mathrm{CN}_{\beta}(r)$. The coordination numbers for a species $\beta$ around a molecule $\alpha$ can be obtained by $\mathrm{CN}_{\beta}(r)=4 \pi \rho_{\beta} \int_{0}^{r} R^{2} g_{\alpha \beta}(R) \mathrm{d} R$ with the number density $\rho_{\beta}$.

The center-of-mass RDFs between TFSI and lithium ions are shown in Fig. 2. More results for different components are depicted in the ESI. $\dagger$ Interestingly, one can see that the RDF between lithium and TFSI strongly depends on the amount of urea in the system. For the 1:1 mixture, a double peak structure in the first coordination shell between $r=0.28 \mathrm{~nm}$ and $0.52 \mathrm{~nm}$ can be observed which can be assigned to monodentate and bidentate coordinated TFSI ions (one or two oxygen atoms of one TFSI ion bound to a single lithium ion). ${ }^{43,44}$ The double peak structure is less pronounced for the $1: 3$ mixture and vanishes completely for the 1:6 mixture. Hence, it can be concluded that TFSI significantly prefers the monodentate coordination at higher urea concentrations. Furthermore, a second coordination peak of TFSI at $r \approx 0.92 \mathrm{~nm}\left(\mathrm{U}_{1: 1}\right)$ is shifted to shorter distances for increasing urea concentrations. With regard to these results, it becomes obvious that a higher urea concentration leads to a more compact coordination of TFSI around lithium ions.

The corresponding findings for the coordination numbers of urea and TFSI molecules around lithium ions are shown in the middle of Fig. 2. It can be seen that urea molecules in comparison to TFSI accumulate at shorter distances around lithium ions, which can be attributed to the smaller size of urea. Moreover, the plateau region of the first urea shell around the lithium ion, which is located at $r=0.3-0.45 \mathrm{~nm}$, is filled with an increasing number of urea molecules at higher urea concentration in order to replace TFSI ions. Hence, while the TFSI number in the first shell decreases, the number of urea molecules increases. These findings explain the occurrence of a monodentate TFSI coordination at higher urea concentration due to a larger number of urea molecules in the local vicinity around lithium ions. With regard to the RDFs for lithium ions (shown in the ESI $\dagger$ ), one can calculate the potential of mean force in accordance with $\operatorname{PMF}(r)=-R T \log \left(g_{\beta}(r) / g_{\beta}(\infty)\right)$ with the molar gas constant $R$ and the temperature $T$ to estimate the
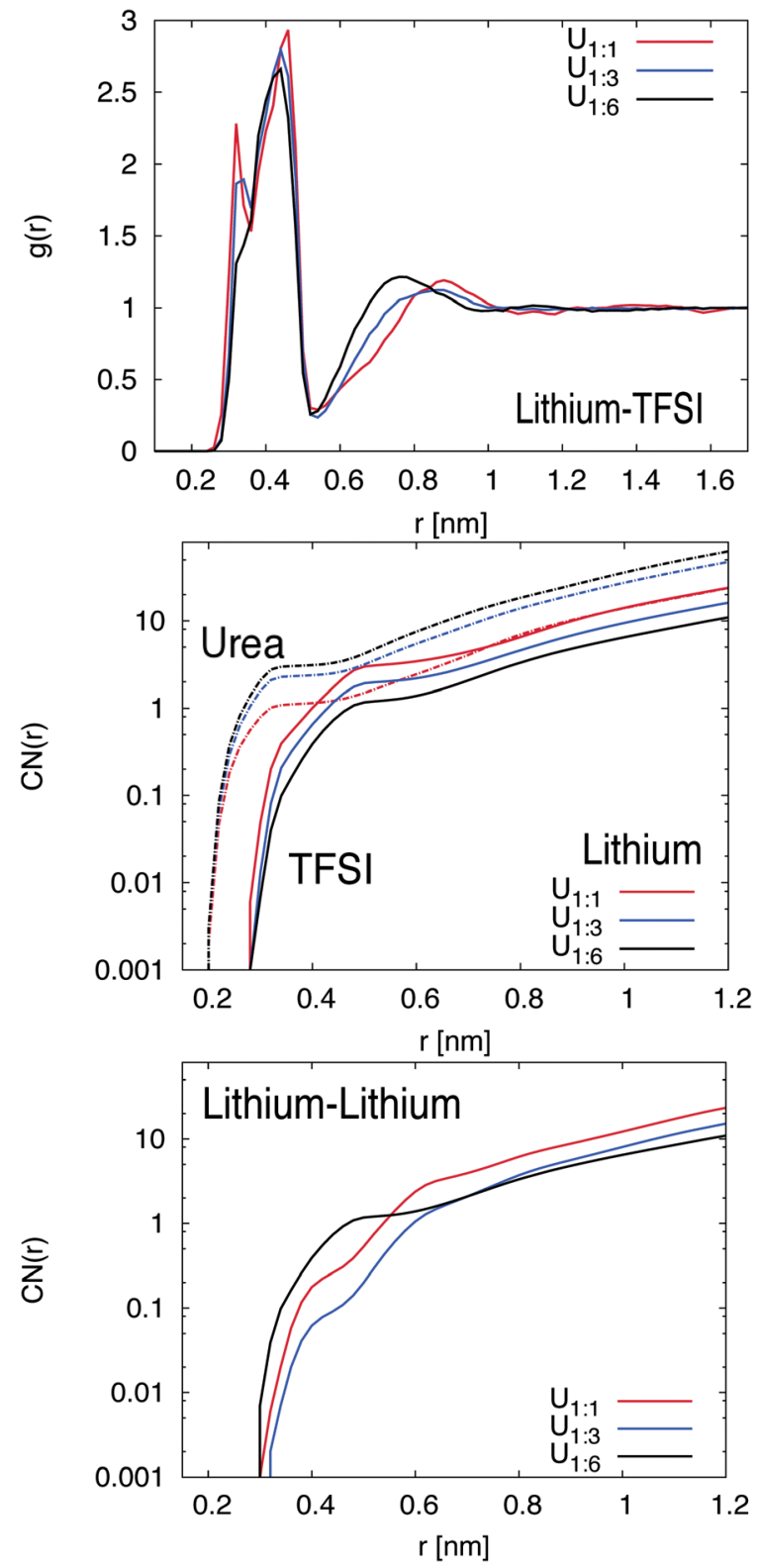

Fig. 2 (top) Center-of-mass radial distribution function $g(r)$ for TFSI around lithium ions for different urea concentrations as denoted in the legend. (middle) Center-of-mass coordination number $\mathrm{CN}(r)$ for urea (slashed lines) and TFSI ions (solid lines) around lithium. (bottom) Center-of-mass coordination number $\mathrm{CN}(r)$ for lithium around lithium ions. The colored lines denote different urea concentrations as described in the legend.

individual binding strength. The resulting values with regard to the binding to lithium ions are $\operatorname{PMF}(r) \approx-3 \mathrm{~kJ} \mathrm{~mol}^{-1}$ for TFSI and $\operatorname{PMF}(r) \approx-4 \mathrm{~kJ} \mathrm{~mol}^{-1}$ for urea, valid for all mixtures in the first coordination shell at $r=0.27 \mathrm{~nm}$. Moreover, the results for the RDF between TFSI and urea indicate only a very weak affinity between urea and TFSI which even decreases at higher urea concentrations. Therefore, we can safely assume that the presence of urea mostly affects the properties of lithium ions in contrast to the previously found strong interaction between urea and negatively charged spheres in aqueous solution. ${ }^{19}$ Based on these findings, we can relate the lithium preference of 
urea to the high dipole moment $(\mu=4.56 \mathrm{D}$ ) of the molecule which favors the oxygen orientation of urea towards lithium ions in good agreement with experimental and numerical findings. ${ }^{14,15}$ It is noteworthy that comparable results for oxygen orientation towards cations were also found for the solvation of sodium ions in dimethyl sulfoxide. ${ }^{45}$ These conclusions are also supported by the results for the local/bulk partition coefficient as shown in the ESI $\dagger$ which reveals the accumulation behavior of different species around a central particle. Based on the corresponding results, it can be concluded that higher urea concentrations enforce a significant replacement of TFSI around lithium ions. The corresponding probabilities for specific coordination numbers of urea and TFSI around lithium ions in the first coordination shell can be also found in the ESI. $\dagger$ In fact, for the lowest urea concentration $\left(\mathrm{U}_{1: 1}\right)$, we found maximum probabilities around $30 \%$ for two and three coordinating TFSI ions which decreases to the values between one and two TFSI ions (both with probabilities around $35 \%)$ for $\mathrm{U}_{1: 3}$ and finally one coordinating TFSI ion with an occurrence probability of roughly $60 \%$ for $U_{1: 6}$. In contrast, the number of coordinating urea molecules increases with higher urea concentration from one molecule with a probability of $65 \%$ for $\mathrm{U}_{1: 1}$ over two molecules (both with probabilities around $37 \%$ ) for $\mathrm{U}_{1: 3}$ to three urea molecules with a maximum probability of $40 \%$ for $\mathrm{U}_{1: 6}$. Thus, we observe a competition between TFSI and urea in occupying the first coordination shell around lithium ions which is in agreement with the roughly comparable potentials of mean force for both species as discussed above. In summary, increasing urea concentrations impose a decreasing TFSI coordination number and favor a monodentate coordination. Moreover, one can assume that different TFSI coordination properties influence the resulting lithium ion transport behavior which was discussed above.

Finally, the influence of urea on the lithium-lithium ion coordination number is shown at the bottom of Fig. 2. It can be clearly seen for distances $r \geq 0.6 \mathrm{~nm}$, that the lithium coordination number for systems with higher urea concentration is significantly smaller. In contrast, for distances shorter than $r=0.6 \mathrm{~nm}$, one can instead observe an increase of the lithium coordination number for higher urea concentrations. In fact, these results clearly illustrate that urea influences the short-range as well as the long-range order around lithium ions. These findings are also supported by the corresponding lithium-lithium radial distribution functions as presented in the ESI. $\dagger$

In order to study the energetic contributions to the binding mechanism in more detail, we calculated the potential energies, namely electrostatic and Lennard-Jones interactions between the constituents of the DEE. The results are depicted in the ESI $\dagger$ and demonstrate that the systems are strongly dominated via electrostatic interactions. In contrast, the influence of Lennard-Jones interactions is negligible. At a low urea content, the system is dominated by strong interactions between lithium and TFSI ions. For increasing urea concentration, the amount of urea binding energy increases whereas the importance of the TFSI interaction with lithium ions decreases. In more detail for the $\mathrm{U}_{1: 3}$ mixture, one can observe that the electrostatic potential energy between lithium and TFSI ions is nearly comparable to lithium ions and urea. These findings point at a critical urea concentration, which is needed to foster the formation of a DEE. ${ }^{14}$ Thus, it can be concluded that the interaction between lithium ions and urea is mainly responsible for the depression of the melting temperature.

In order to understand the influence of the initial local environment, we studied the influence of coordinating TFSI ions on the dynamic behavior of $\mathrm{Li}^{+}$. Therefore, we determined the number of neighboring TFSI ions in the first shell around lithium for the systems $U_{1: 3}$ and $U_{1: 6}$ at different points of the simulation and considered them as starting points for our analysis. The corresponding evolution in time provides the MSD for the central lithium ion and reveals the influence of the initial local environment configuration on the lithium dynamics. Our results evidence that the initial number of TFSI ions $\mathrm{NB}_{\text {TFSI }}$ in the first coordination shell around $\mathrm{Li}^{+}$strongly influences the dynamic behavior of lithium ions (Fig. 3). Based on our findings, it can be concluded that a larger number of coordinating TFSI ions $\mathrm{NB}_{\text {TFSI }} \geq 4$ strongly decrease the lithium MSD at short times while the influence for $\mathrm{NB}_{\mathrm{TFSI}} \leq 3$ is less pronounced. In agreement with previous results (Fig. 1), we found a slightly higher MSD for lithium ions in the presence of higher urea concentrations (bottom part of Fig. 3). Moreover, it can be observed that the onset of the diffusive regime occurs at shorter times for a smaller number of initial coordinating TFSI ions. However, the relative slow down for a fixed number of coordinating TFSI ions $\mathrm{NB}_{\mathrm{TFSI}}$ between both mixtures is roughly comparable. At long times, one can clearly see that all results coincide with a single MSD, which indicates that the influence of an initial local environment becomes negligible on these time scales. With regard to these findings, we conclude that higher TFSI coordination numbers significantly decrease the lithium transference numbers at short times in agreement with some properties observed for ionic liquids. ${ }^{12,43}$ In addition, our results indicate that higher concentrations of urea impose a faster MSD for all species.

In order to investigate the exchange of TFSI ions in the first coordination shell in more detail, we calculated the autocorrelation function for the number of TFSI ions around lithium ions $\left\langle\mathrm{NB}_{\mathrm{TFSI}}(t) \mathrm{NB}_{\mathrm{TFSI}}\left(t_{0}\right)\right\rangle /\left\langle\mathrm{NB}_{\mathrm{TFSI}}{ }^{2}\left(t_{0}\right)\right\rangle$ within $r=0.27 \mathrm{~nm}$. The corresponding results for the autocorrelation function are shown in the ESI. $\dagger$ For a 1:1 mixture, we found the slowest decay of the autocorrelation function with the largest residence time of $\tau \approx 15 \mathrm{~ns}$. Increasing the concentration of urea leads to a fast decrease of the residence times $\left(\tau \approx 2.7 \mathrm{~ns}\left(\mathrm{U}_{1: 3}\right)\right.$ and $\left.\tau \approx 0.6 \mathrm{~ns}\left(\mathrm{U}_{1: 6}\right)\right)$ and thus a faster exchange rate of TFSI molecules. These results are in good agreement with the data presented in the bottom panel of Fig. 3, where it can be seen that the corresponding onset of diffusive behavior at $\tau_{\mathrm{D}}$ follows the relation $\tau_{\mathrm{D}}>\tau$, which reveals that the rearrangement of the local coordination shell is of significant importance for the dynamic behavior of $\mathrm{Li}^{+}$. Thus, a larger amount of urea favors the occurrence of higher TFSI exchange rates which can be brought into agreement with a transition from vehicular to structural transport of lithium ions. ${ }^{12}$ 


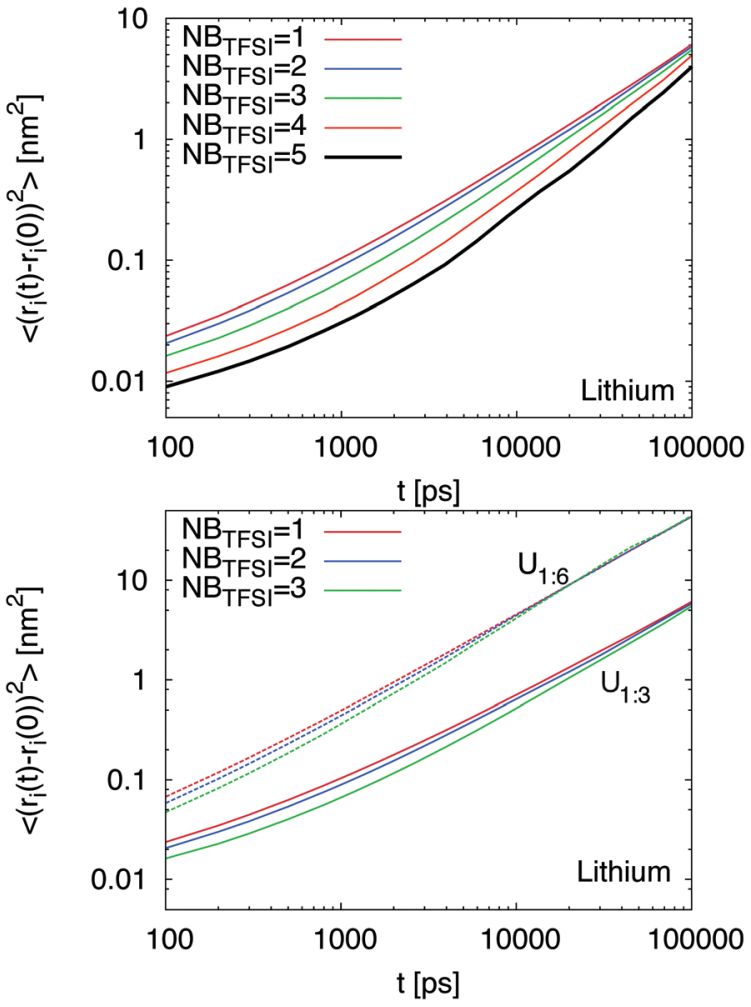

Fig. 3 (top) Mean square displacement of $\mathrm{Li}^{+}$depending on the number of TFSI molecules $N_{\text {TFSI }}$ in the first coordination shell for a mixture $U_{1: 3}$. (bottom) Comparison of $\mathrm{Li}^{+}$-MSD for $\mathrm{U}_{1: 3}$ (solid lines) and $\mathrm{U}_{1: 6}$ (dashed lines) in terms of the number of coordinated TFSI molecules NB $\mathrm{NFSI}_{\mathrm{TFS}} \leq 3$.

In summary, the results of our atomistic molecular dynamics simulations reveal a strong influence of urea on the local environment around $\mathrm{Li}^{+}$. Previous experimental studies showed that LiTFSI/urea mixtures form deep eutectic electrolytes at specific molar ratios. ${ }^{14}$ We found pronounced effects of urea on the transport and the structural properties of LiTFSI. Based on our findings, we can conclude that urea systematically replaces TFSI ions in the first coordination shell around lithium ions which results in the formation of a DEE and furthermore induces a faster diffusional behavior. Hence, we assume that LiTFSI/urea mixtures are potential candidates to improve the ion transport in lithium metal and lithium ion batteries. In the future, we will study these systems by experiments and simulations in more detail to verify their applicability for electrochemical storage devices.

The authors thank Markus Blank-Burian and Oliver Rubner for technical help and the Deutsche Forschungsgemeinschaft (DFG) via the cluster of excellence 'Simulation Technology' (EXC 310) for financial support.

\section{References}

1 Q. Zhang, K. D. O. Vigier, S. Royer and F. Jérôme, Chem. Soc. Rev., 2012, 41, 7108-7146.

2 B. Tang and K. H. Row, Monatsh. Chem., 2013, 144, 1427-1454.
3 D. A. Alonso, A. Baeza, R. Chinchilla, G. Guillena, I. M. Pastor and D. J. Ramón, Eur. J. Org. Chem., 2016, 4, 612-632.

4 A. P. Abbott, G. Capper, D. L. Davies, R. K. Rasheed and V. Tambyrajah, Chem. Commun., 2003, 70-71.

5 H. Sun, Y. Li, X. Wu and G. Li, J. Mol. Model., 2013, 19, 2433-2441.

6 A. Romero, A. Santos, J. Tojo and A. Rodriguez, J. Hazard. Mater., 2008, 151, 268-273.

7 V. Lesch, A. Heuer, V. A. Tatsis, C. Holm and J. Smiatek, Phys. Chem. Chem. Phys., 2015, 17, 26049-26053.

8 A. D. Pauric, I. C. Halalay and G. R. Goward, Phys. Chem. Chem. Phys., 2016, 18, 6657-6667.

9 H. Ye, J. Huang, J. J. Xu, A. Khalfan and S. G. Greenbaum, J. Electrochem. Soc., 2007, 154, A1048-A1057.

10 M. Ishikawa, T. Sugimoto, M. Kikuta, E. Ishiko and M. Kono, J. Power Sources, 2006, 162, 658-662.

11 O. Borodin, G. D. Smith and W. Henderson, J. Phys. Chem. B, 2006, 110, 16879-16886.

12 Z. Li, G. D. Smith and D. Bedrov, J. Phys. Chem. B, 2012, 116, 12801-12809.

13 A. Matic and B. Scrosati, MRS Bull., 2013, 38, 533-537.

14 H. Liang, H. Li, Z. Wang, F. Wu, L. Chen and X. Huang, J. Phys. Chem. B, 2001, 105, 9966-9969.

15 S. Li, L. Liu, Z. Cao, J.-Q. Wang and T.-Y. Yan, Acta Phys. Chim. Sin., 2007, 23, 983-986.

16 L. Sim, R. Yahya and A. Arof, Opt. Mater., 2016, 56, 140-144.

17 Y. Hu, Z. Wang, H. Li, X. Huang and L. Chen, Spectrochim. Acta, Part A, 2005, 61, 2009-2015.

18 D. Horinek and R. R. Netz, J. Phys. Chem. A, 2011, 115, 6125-6136.

19 J. Smiatek, J. Phys. Chem. B, 2014, 118, 771-782.

20 A. Narayanan Krishnamoorthy, C. Holm and J. Smiatek, J. Phys. Chem. B, 2014, 118, 11613-11621.

21 F. Rodriguez-Ropero and N. F. A. van der Vegt, Phys. Chem. Chem. Phys., 2015, 17, 8491-8498.

22 S. Micciulla, J. Michalowsky, M. A. Schroer, C. Holm, R. von Klitzing and J. Smiatek, Phys. Chem. Chem. Phys., 2016, 18, 5324-5335.

23 H.-R. Jhong, D. S.-H. Wong, C.-C. Wan, Y.-Y. Wang and T.-C. Wei, Electrochem. Commun., 2009, 11, 209-211.

24 L. Vieira, A. Whitehead and B. Gollas, J. Electrochem. Soc., 2014, 161, D7-D13.

25 W. L. Jorgensen, D. S. Maxwell and J. Tirado-Rives, J. Am. Chem. Soc., 1996, 118, 11225-11236.

26 H. J. C. Berendsen, D. van der Spoel and R. van Drunen, Comput. Phys. Commun., 1995, 91, 43-56.

27 D. van der Spoel, E. Lindahl, B. Hess, G. Groenhof, A. E. Mark and H. J. C. Berendsen, J. Comput. Chem., 2005, 26, 1701-1718.

28 S. Pronk, S. Páll, R. Schulz, P. Larsson, P. Bjelkmar, R. Apostolov, M. R. Shirts, J. C. Smith, P. M. Kasson, D. van der Spoel, B. Hess and E. Lindahl, Bioinformatics, 2013, 29, 845-854.

29 J. M. Martinez and L. Martinez, J. Comput. Chem., 2003, 24, 819-825. 
30 L. Martinez, R. Andrade, E. G. Birgin and J. M. Martinez, J. Comput. Chem., 2009, 30, 2157-2164.

31 T. Mendez-Morales, J. Carrete, S. Bouzon-Capelo, M. PerezRodriguez, O. Cabeza, L. J. Gallego and L. M. Varela, J. Phys. Chem. B, 2013, 117, 3207-3220.

32 F. Dommert, K. Wendler, R. Berger, L. Delle Site and C. Holm, ChemPhysChem, 2012, 13, 1625-1637.

33 V. Lesch, A. Heuer, C. Holm and J. Smiatek, Phys. Chem. Chem. Phys., 2015, 17, 8480-8490.

34 V. Lesch, A. Heuer, C. Holm and J. Smiatek, ChemPhysChem, 2016, 387-394.

35 G. Bussi, D. Donadio and M. Parrinello, J. Chem. Phys., 2007, 126, 014101.

36 H. Berendsen, J. Postma, W. van Gunsteren, A. DiNola and J. Haak, J. Chem. Phys., 1984, 81, 3684-3690.

37 B. Hess, H. Bekker, H. J. C. Berendsen and J. G. E. M. Fraaije, J. Comput. Chem., 1997, 18, 1463-1472.
38 T. Darden, D. York and L. Pedersen, J. Chem. Phys., 1993, 98, 10089-10092.

39 U. Essmann, L. Perera, M. L. Berkowitz, T. Darden, H. Lee and L. G. Pedersen, J. Chem. Phys., 1995, 103, 8577-8593.

40 A. Einstein and M. von Smoluchowski, Untersuchungenüber die Theorie der Brownschen Bewegung, Deutsch, 1997.

41 F. Dommert, J. Schmidt, C. Krekeler, Y. Y. Zhao, R. Berger, L. Delle Site and C. Holm, J. Mol. Liq., 2010, 152, 2-8.

42 V. Lesch, S. Jeremias, A. Moretti, S. Passerini, A. Heuer and O. Borodin, J. Phys. Chem. B, 2014, 118, 7367-7375.

43 V. Lesch, Z. Li, D. Bedrov, O. Borodin and A. Heuer, Phys. Chem. Chem. Phys., 2016, 18, 382-392.

44 J. Pitawala, A. Martinelli, P. Johansson, P. Jacobsson and A. Matic, J. Non-Cryst. Solids, 2015, 407, 318-323.

45 J. Smiatek, A. Wohlfarth and C. Holm, New J. Phys., 2014, 16, 025001. 